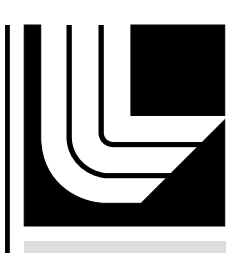

LAWRENCE LIVERMORE NATIONAL LABORATORY

\title{
Crystallographic Anisotropy of Wear on a Polycrystalline Diamond Surface
}

B. El-dasher, J. J. Gray, J. W. Tringe, C. Wild, E. Wörner, P. Koidl, J. Biener, A. V. Hamza

November 23, 2005

Applied Physics Letters 
This document was prepared as an account of work sponsored by an agency of the United States Government. Neither the United States Government nor the University of California nor any of their employees, makes any warranty, express or implied, or assumes any legal liability or responsibility for the accuracy, completeness, or usefulness of any information, apparatus, product, or process disclosed, or represents that its use would not infringe privately owned rights. Reference herein to any specific commercial product, process, or service by trade name, trademark, manufacturer, or otherwise, does not necessarily constitute or imply its endorsement, recommendation, or favoring by the United States Government or the University of California. The views and opinions of authors expressed herein do not necessarily state or reflect those of the United States Government or the University of California, and shall not be used for advertising or product endorsement purposes. 
UCRL-JRNL-217284

\title{
Crystallographic Anisotropy of Wear on a Polycrystalline Diamond Surface
}

B. S. El-dasher, J. J. Gray, J. W. Tringe, C. Wild, E. Wörner, P. Koidl, J. Biener and A. V. Hamza

\begin{abstract}
We correlate topography and diffraction measurements to demonstrate that grain orientation profoundly influences polishing rates in polycrystalline diamond synthesized by chemical vapor deposition. Grains oriented with $\{111\}$ or $\{100\}$ planes perpendicular to the surface normal polish at significantly lower rates compared with grains of all other orientations when the surface is polished in continuously varying in-plane directions. These observations agree with predictions of the periodic bond chain vector model, developed previously for single crystals, and indicate that the polishing rate depends strongly on the number of PBC vectors that are within $10^{\circ}$ of the exposed surface plane.
\end{abstract}


The invention of chemical vapor-deposited (CVD) diamond has revolutionized the diamond industry by lowering the cost and vastly increasing the size and shape of structures that can be coated with diamond, allowing entirely new applications for this hardest of all materials. Polycrystalline CVD diamond films can be deposited on a variety of substrates over large (100's of $\mathrm{cm}^{2}$ ) areas, and the thermal and mechanical properties of these films are similar to those of natural diamond. ${ }^{1-11}$. One of the main obstacles to many potential diamond applications is the roughness of the as-deposited films. Consequently, the surface often needs to be polished for applications such as high-performance optical components, cutting surfaces and heat-sinks, where a lower degree of roughness translates directly to improved performance. Unfortunately, polycrystalline diamond is very difficult to polish due to highly anisotropic polishing behavior: typical wear rates can vary by up to two orders of magnitude depending on both crystal orientation and polishing direction. ${ }^{12}$ In this work, we introduce a simple and straightforward method to assess the anisotropic polishing behavior of polycrystalline diamond by analyzing the correlation between grain orientation and surface topography. The relative polishing rate obtained by this method can be explained by the number of periodic bond chain (PBC) vectors affecting the polished surface.

The polycrystalline diamond sample studied in this work was grown on a Si substrate using microwave plasma-enhanced chemical vapor deposition (PECVD). The substrate was chemically etched away, yielding a freestanding specimen approximately $1 \mathrm{~mm}$ thick. The growth surface was then polished to a nanometer (RMS roughness) finish using a high-speed grinding wheel with diamond grit as abrasive. During polishing, the specimen was continuously rotated to minimize polishing anisotropy. A 5mm diameter disk was then laser-cut for the experiments reported here.

Surface orientation and surface topography were studied by electron backscatter diffraction (EBSD) and atomic force microscopy (AFM). The sample surface morphology was imaged 
using a Digital instruments Inc., Dimension 3100 Scanning Probe Microscope (Santa Barbara, CA) in contact mode at a sampling rate of $1 \mathrm{~Hz}$. Large (100 by $100 \mu \mathrm{m}^{2}$ ) AFM images were acquired on a grid pattern to map the same regions as those scanned by EBSD. Additionally, smaller ( 5 by $5 \mu \mathrm{m}^{2}$ ) images were acquired to examine the surface morphology dependence on grain orientation. Two reference marks on opposite sides of the specimen allowed for spatial matching between the two techniques, and hence the correlation of the crystallographic orientation with topography. The advantage of the method is that it allows for a direct comparison of the wear behavior of different orientations since all orientations were processed simultaneously under identical conditions.

The polishing behavior of diamond is highly anisotropic as revealed by the correlation between grain orientation and surface topography. This is illustrated by Figure 1 which shows the location of one of the EBSD scan areas relative to the fiducial marks, as well as the correlation between orientation and AFM topography. \{111\}-oriented grains were consistently the highest grains observed in any 100 by $100 \mu \mathrm{m}^{2}$ area, as illustrated in Figures 1 (c) and 1 (d). The lightest grain in the EBSD scan 1(c), representing the grain closest to $\{111\}$, corresponds to the lightest grain in Figure 1(d), representing the highest grain in the AFM scan. Because this was a consistently observed phenomenon, all subsequent analysis was performed with grain depths determined relative to $\{111\}$-oriented grains.

Specifically, we assume that all grains with an orientation within $5^{\circ}$ of $\{111\}$ polish at the same rate; these are defined as reference grains, and eleven grains out of the 1000-grain data set met this criterion. Since each reference grain was neighbored by four to six other grains, a total of 48 relative depths were measured. These relative depths were binned into three sets based on the extent of relative wear: low (0 to $10 \mathrm{~nm})$, medium (10 to $40 \mathrm{~nm})$, and high ( $>40 \mathrm{~nm})$. Figure 2 displays the relative wear rates as a function of grain orientation in the standard stereographic 
triangle. Grains oriented near $\{111\}$ are the highest, and thus the most resistant to polishing wear. This is not surprising since the $\{111\}$ planes have the highest atomic density. Interestingly, however, grains oriented near $\{001\}$ are also among the most wear resistant. On the other hand, the grains in between the $\{111\}$ and $\{001\}$ corners possess intermediate wear resistance, and those nearer $\{101\}$ are generally the least wear resistant. Although wear rate and grain depth are correlated, we note that grain depth is not a direct measure of wear rate since areas of lower wear are in a much closer contact to the grinding surface compared to recessed areas.

To interpret these results, we use the periodic bond chain (PBC) vector model, recently applied by van Bouwelen and van Enckevort to describe the anisotropic polishing rate as function of crystallographic orientation for single crystal diamond surfaces. ${ }^{13}$ This simple, yet powerful, model defines the PBC as an uninterrupted chain of bonded growth units connecting two translation-equivalent lattice positions in the crystal. For diamond, there are six PBC chains corresponding to the six $<110>$ symmetrically equivalent directions. Taking the vector product of $F$, the direction of the polishing force, and $P$, the direction of the nearest $\mathrm{PBC}$ vector, the model predicts difficult polishing for $F \cdot P \geq \sim 0.9$ and easy polishing for $F \cdot P \leq \sim 0.7$ when both $F$ and $P$ are defined as unit vectors. These predictions are consistent with observed crystallographic polishing anisotropy for single diamond crystals. ${ }^{13}$

The PBC model predicts that $<110>$-type directions in diamond are most wear-resistant because they possess uninterrupted chains of bonded growth units. For a given crystallographic direction, resistance to wear is therefore maximized when the polishing direction is parallel to one of the PBC vectors. Using this model, we can partially explain the observed height distribution if we assume that the wear resistance of a given crystal plane is proportional to the number of inplane PBC vectors. $\{111\}$ diamond planes contain three $<110>$ vectors, $\{001\}$ planes contain two $<110>$ vectors, and $\{101\}$ planes contain one $<110>$ vector. However, the average of the absolute value of $F \cdot P$ product, taken for all in-plane polishing directions fails to predict the observed 
wear-resistance of $\{001\}$ oriented grains. The average for $\{001\}$ grains is 0.91 , as different from the $\{111\}$ average of 0.95 as it is from the $\{101\}$ value of 0.87 . Based solely on average $F \cdot P$ then, an intermediate polishing resistance is expected for $\{001\}$-oriented grains.

To understand the reason for the higher wear resistance for $\{001\}$-oriented grains in Fig. 2, the full in-plane angular dependence of the $F \cdot P$ product must be examined. In Figure 3(a), we plot the magnitude of the $F \cdot P$ product as a function of misorientation angle from a $<110>$ vector in one of each of the three principle planes. The behavior of the $F \cdot P$ magnitude for the (111) and (001) planes is quite similar, with values appreciably higher than for the (101) plane. In fact, for $\{101\}$ grains there are a significant number of polishing directions for which the grains are expected to polish very efficiently. It is interesting to note however that there are directions $(<112>$ type) in which resistance to polishing increases due to the effect of out of plane PBC vectors. However, it is likely then that for these $\{101\}$ grains the overall polishing rate is dominated by the fast polishing that occurs when the grains are oriented with respect to the polishing direction that minimizes $|\boldsymbol{F} \cdot \boldsymbol{P}|$.

The PBC vector model can predict the relative crystallographic anisotropy of polishing wear if we assume that the wear rate is dominated by the PBC vectors nearest to the polishing direction. In this analysis, PBC vectors are considered influential to the polishing rate of the grains only if they are within $10^{\circ}$ of the specimen surface plane. We base this value on experimental evidence for the effect of tilt on polishing rate, and on our experimental limits for measuring grain orientation. For example, for single crystals, experiments have shown that tilting away from a hard polishing direction by as little as $5^{\circ}$ increases the wear rate by more than an order of magnitude. ${ }^{14}$ Further, our definition of a “ $\{111\}$ ” or “ $\{100\}$ ” grain is a grain whose true orientation is within $5^{\circ}$ of these plane normals. In Figure 3(b) then, we correlate the average depth of the 48 grains studied with the number of $\mathrm{PBC}$ vectors influencing their respective 
polishing wear, and it can be seen that the average grain depth clearly scales with the inverse of the number of PBC vectors influencing the grains. This strong dependence of wear rate on the number of influential PBC vectors constitutes the most important and convincing experimental evidence for the PBC polishing model in diamond. Although our study focused on diamond, these results may be much more broadly applicable: PBC vectors are present in every polycrystalline material, and will similarly affect local polishing rates in a wide range of systems.

Acknowledgement This work was performed under the auspices of the US Dept. of Energy by the Univ. of California Lawrence Livermore National Lab under Contract W-7405-ENG-48. 

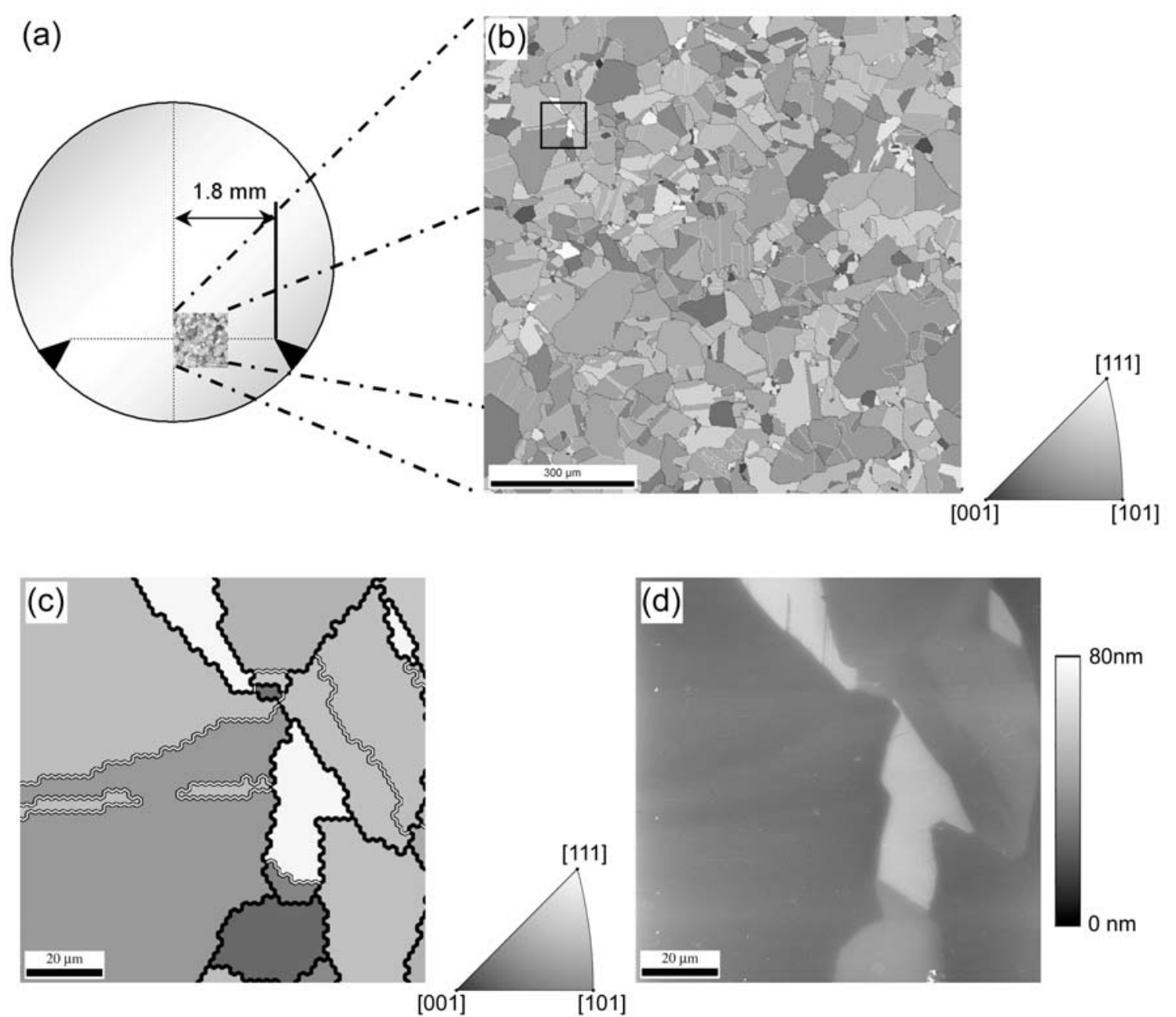

Figure 1. (a) Schematic of polished diamond specimen showing one of two $1 \mathrm{~mm}^{2}$ areas of interest, (b) Electron Backscatter Diffraction Map showing the grains mapped in the indicated $1 \mathrm{~mm}^{2}$ area, with the grayscale levels indicating the misorientation angle from [111]. The black box represents a $100 \mu \mathrm{m}$ by $100 \mu \mathrm{m}$ area, shown in (c) as an EBSD map, and in (d) as an atomic force microscopy map. Comparison of (c) and (d) shows differing grain height as a function of orientation. 


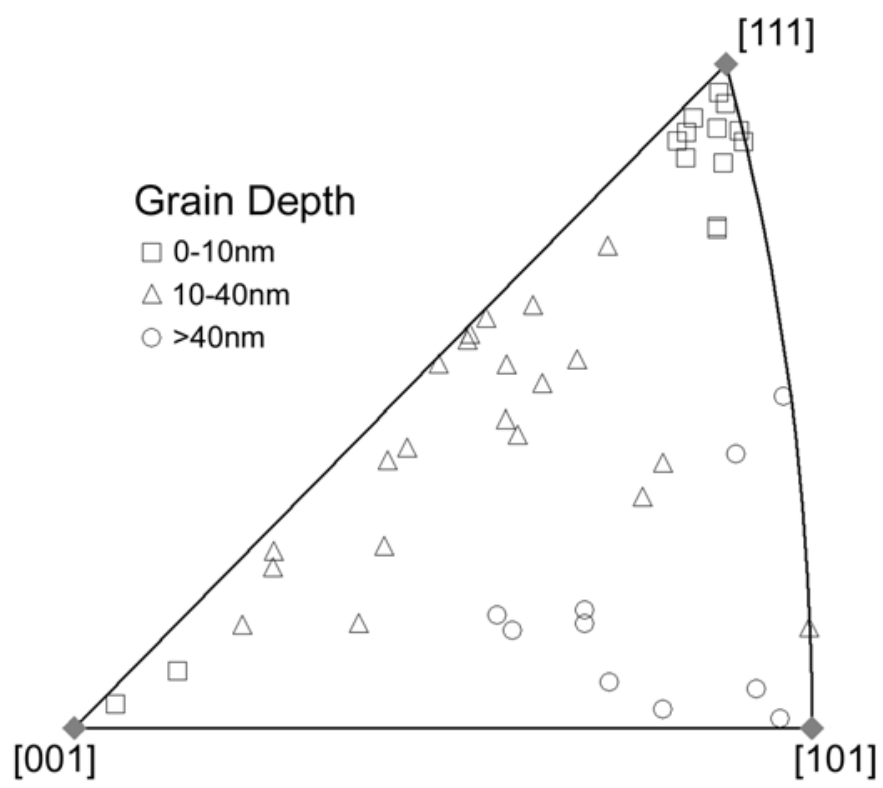

Figure 2. Anisotropy of diamond polishing: Plot of the grain depth as a function of plane normal in the standard stereographic triangle relative to the nearest $\{111\}$ grain, showing the effect of grain crystallography on the relative polishing rate. 
(a)

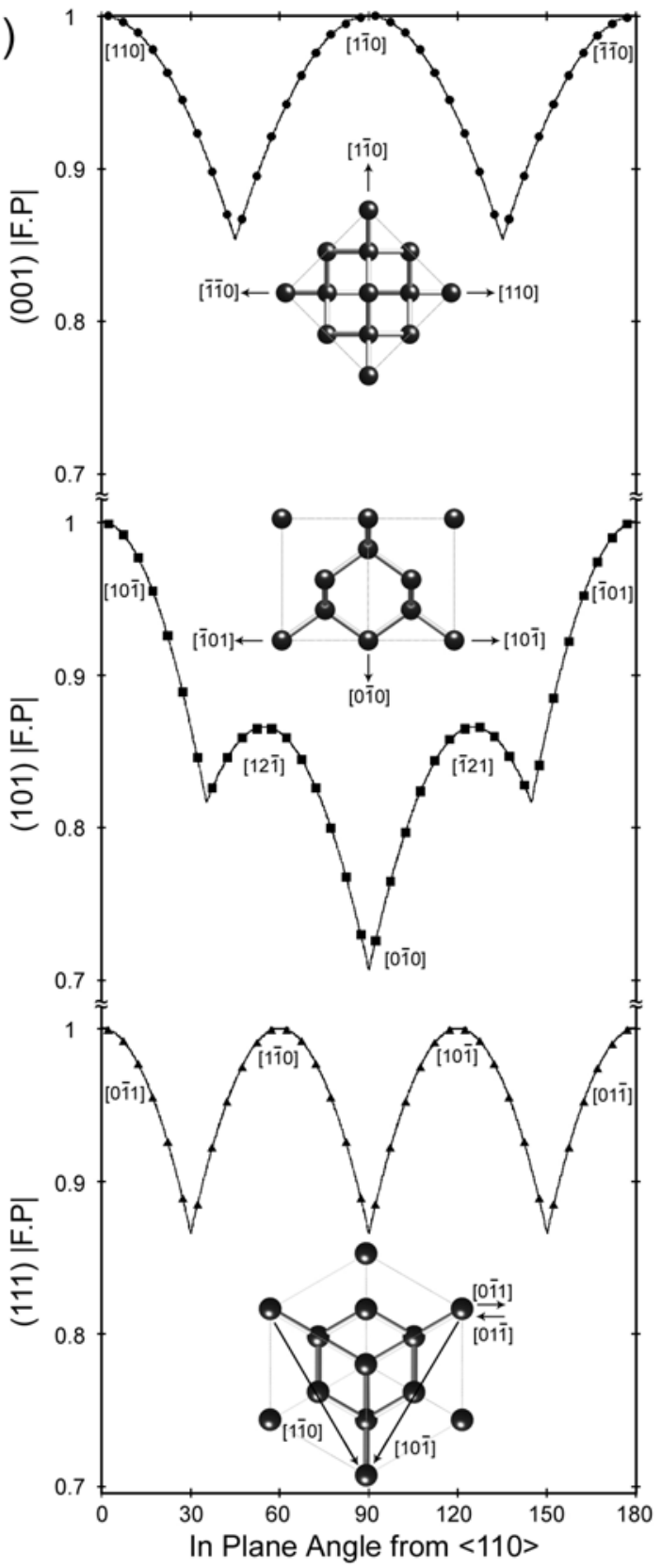

(b)

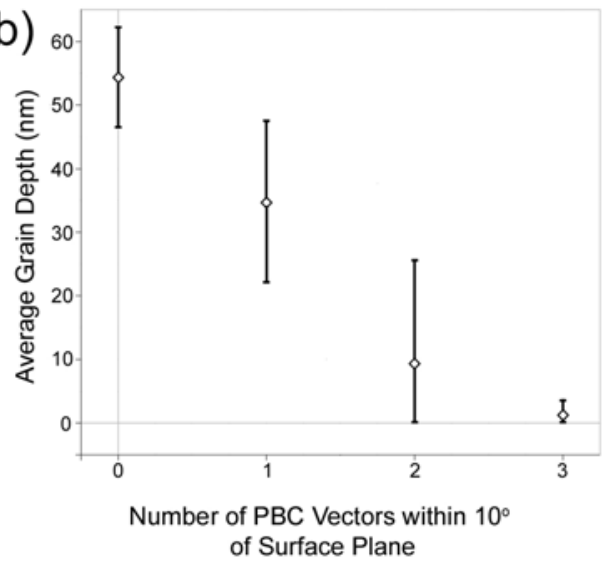

Figure 3. (a) Calculated values of the FP product as a function of in-plane rotation of $F$ for the (001), (101) and (111) planes. At $0^{\circ}, F$ is a $<110>$ direction. Inset unit cell projections correlate in-plane vectors to plot features. Note that out-of-plane $P$ vectors influence the FP product for the $\{101\}$ polishing plane. (b) The effect of the number of $\mathrm{PBC}$ vectors within $10^{\circ}$ of the polishing plane on the amount of material polished for all the grains studied, illustrating the increase in polishing difficulty due to the influence of PBC vectors. 


\section{References}

1 V. Ralchenko, L. Nistor, E. Pleuler, A. Khomich, I. Vlasov, and R. Khmelnitskii, Diamond and Related Materials 12, 1964-1970 (2003).

2 D. J. Pickrell, K. A. Kline, and R. E. Taylor, Applied Physics Letters 64, 23532355 (1994).

3 R. Kuschnereit, P. Hess, D. Albert, and W. Kulisch, Thin Solid Films 312, 66-72 (1998).

$4 \quad$ S. E. Grillo and J. E. Field, Wear 254, 945-949 (2003).

5 L. Fayette, B. Marcus, M. Mermoux, G. Tourillon, K. Laffon, P. Parent, and F. Le Normand, Physical Review B 57, 14123-14132 (1998).

6 B. Bhushan, V. V. Subramaniam, A. Malshe, B. K. Gupta, and J. Ruan, Journal of Applied Physics 74, 4174-4180 (1993).

7 P. Puech, S. Pinel, R. G. Jasinevicius, and P. S. Pizani, Journal of Applied Physics 88, 4582-4585 (2000). J. A. Weima, R. Job, W. R. Fahrner, G. C. Kosaca, N. Muller, and T. Fries, Journal of Applied Physics 89, 2434-2440 (2001).

9 H. D. Espinosa, B. Peng, B. C. Prorok, N. Moldovan, O. Auciello, J. A. Carlisle, D. M. Gruen, and D. C. Mancini, Journal of Applied Physics 94, 6076-6084 (2003).

S. Miyake, Applied Physics Letters 65, 1109-1111 (1994).

R. L. C. Wu, A. K. Rai, and K. Miyoshi, Applied Physics Letters 68, 1054-1056 (1996).

12 S. E. Grillo and J. E. Field, Wear 211, 30-34 (1997).

F. M. van Bouwelen and W. J. P. van Enckevort, Diamond and Related Materials 8, 840-844 (1999). Physics 33, 985-990 (2000). 